\title{
ANÁLISIS FONÉTICO DE LOS PATRONES MELÓDICOS LOCALES EN ESPAÑOL: PATRONES ACENTUALES ${ }^{1}$
}

\author{
JUAn María Garkido Almiñana \\ Universidad Pompeu Fabra \\ Fundació Barcelona Media Centre d'Innovació
}

\section{RESUMEN}

El objetivo de este trabajo es aportar nuevos datos a la descripción de los patrones acentuales (movimientos melódicos relacionados con el acento) del español desde una perspectiva fonética, a partir del análisis de una muestra amplia de habla realizado con la herramienta automática de estilización, anotación y modelización de contornos melódicos descrita en Garrido 2010, que asume el modelo de representación y modelización de contornos propuesto en Garrido 1996, 2001. El material de habla analizado en este trabajo está formado por un conjunto de grupos acentuales extraído de cuatro corpus diferentes, grabado cada uno por un locutor profesional diferente, tres femeninos y uno masculino, todos ellos hablantes de español peninsular. El total de grupos analizados ascendió a 33.730. Todo este material fue procesado con la herramienta automática para obtener un listado de los patrones melódicos detectados en los grupos acentuales analizados, con una indicación del número de apariciones de cada uno. En el apartado de resultados se describe la forma acústica de los patrones más frecuentes en los grupos acentuales iniciales e interiores de grupo entonativo, de forma separada para cada categoría. Finalmente, en el apartado de conclusiones se analiza la relación de los resultados obtenidos con el estado actual de la descripción de los patrones acentuales del español.

Palabras clave: fonética acústica, entonación, melodía, patrones melódicos, acento, español.

\section{ABSTRACT}

The aim of this work is to offer new data to the description of the stress patterns (pitch movements related to stress) of Spanish from a phonetic point of view, using the analysis of a large sample of speech carried out with the automatic tool of stylisation, annotation and modelisation of pitch contours described in Garrido 2010, which assumes the representation and modeling framework proposed in Garrido 1996,2003 . The analyzed speech material is made out of a set of stress groups obtained from four different corpora, each one recorded by a different professional

\footnotetext{
${ }^{1}$ Este trabajo se ha llevado a cabo con el apoyo económico del programa Ramón y Cajal del Ministerio de Ciencia e Innovación. Mi agradecimiento también a Yesika Laplaza, Montserrat Marquina, Sílvia Rustullet y Junming Yao, por su lectura crítica de este artículo.
} 
speaker, three female and one male, all of them native speakers of Peninsular Spanish. The total amount of analyzed groups is 33.730 . All this material was processed with the automatic tool to obtain a listing of the pitch patterns detected in the analyzed stress groups, with an indication of the counting of each one. In the results section the acoustic shape of the most frequent patterns for the initial and middle stress group categories is described. Finally, in the conclusions section the relation between the obtained results and the state of the art in the description of the pitch patterns related to stress in Spanish is analyzed.

Key Words: acoustic phonetics, intonation, pitch, pitch patterns, stress, Spanish.

RECIBIDO: 19/01/2011

APROBADO: 17/02/2012

\section{INTRODUCCIÓN}

La descripción detallada del inventario de los patrones melódicos relacionados con el acento en español (patrones acentuales, o «acentos tonales» en la tradición fonológica autosegmental, también denominado pretonema en determinadas aproximaciones) ha sido y sigue siendo objeto reiterado de estudio, tanto desde una perspectiva fonética como fonológica, pero está aún lejos de haberse completado. El objetivo de este trabajo es aportar nuevos datos a la descripción de los patrones acentuales del español desde una perspectiva fonética, a partir del análisis de una muestra amplia de habla realizado con la herramienta automática de estilización, anotación y modelización de contornos melódicos descrita en Garrido 2010, que asume el modelo de representación y modelización de contornos propuesto en Garrido 1996, 2001.

En este trabajo se asume, siguiendo la propuesta de Garrido 2001, que el ámbito natural de los patrones acentuales, al menos en español, es el grupo acentual (GA), compuesto por una sílaba tónica y todas las átonas que le siguen hasta la siguiente sílaba tónica o el final del grupo entonativo que lo contiene. El objetivo es describir lo más detalladamente posible la forma de los contornos melódicos que se dan en este ámbito en el corpus de GA analizados, y establecer un inventario de los patrones más frecuentes. Tal como se argumenta en Garrido 2001, la motivación para el uso de esta unidad como unidad descriptiva de los patrones acentuales es básicamente fonética: el inicio del movimiento ascendente-descendente asociado típicamente con la expresión del acento léxico (el «pico» acentual) coincide habitualmente con el comienzo de una sílaba tónica (Fant 1984; Garrido 1996), por lo que estos movimientos tonales abarcarían desde el inicio de una sílaba tónica hasta el inicio de la siguiente. El GA se ha empleado también para la descripción de los movimientos tonales de otras lenguas (Thorsen 1978), y es similar a otras unidades definidas en el ámbito de la fonología prosódica, 
como el pie (Nespor y Vogel 1986) o el sintagma acentual (Beckman y Pierrehumbert 1986; Jun y Fougeron 2000): ambas se definen, al igual que el GA, como unidades formadas por una sílaba tónica (o fuerte) y un número indeterminado de sílabas átonas (o débiles), en las que la sílaba fuerte puede aparecer tanto al principio como al final, según la lengua; el sintagma acentual, además, se define como el ámbito natural de un determinado movimiento o configuración tonal (un acento tonal), como ocurre con el GA, y en la formulación de Jun y Fougeron 2000, las sílabas pueden pertenecer a palabras distintas, también al igual que el GA. Esta unidad, sin embargo, es algo diferente del concepto de «grupo tónico», que Navarro 1944 propuso, con criterios fundamentalmente sintáctico-semánticos, como unidad de ámbito inferior al grupo entonativo, y que tuvo un relativo éxito en estudios posteriores sobre la entonación hispánica.

Estudios anteriores, realizados en el marco del modelo autosegmental (Sosa 1995; Beckman y otros 2002; Fernández y Martínez 2003; Toledo 2000, 2001, 2003, 2004; Estebas y Prieto 2008, entre otros) ofrecen propuestas semejantes de inventarios, algunos también con datos de frecuencia. Este trabajo pretende complementar estos estudios aportando datos, obtenidos a partir del análisis automático de un corpus amplio de grupos acentuales, sobre la forma de sus contornos y sobre el anclaje de sus diferentes inflexiones con las sílabas del GA (el denominado alineamiento tonal).

En el apartado 2 se presenta el estado actual de la descripción de los patrones acentuales en español, y a continuación, en el apartado 3, se presentan los datos de nuestro trabajo. Finalmente, en el apartado de conclusiones, se analizan los resultados obtenidos y se comparan con los estudios anteriores.

\section{Patrones acentuales en español: estado de la CUestión}

Desde las primeras descripciones de los contornos melódicos del español (Navarro 1944, por ejemplo) se ha señalado que la expresión melódica del acento en español se caracteriza por la presencia de un ascenso tonal en los alrededores de las sílabas acentuadas (los llamados «picos de F0»). La alineación tonal de este movimiento ascendente con la sílaba tónica, sin embargo, es aún objeto de controversia.

Ya el propio Navarro 1944 señalaba que el final del movimiento ascendente puede retrasarse de la sílaba tónica a la postónica, fenómeno confirmado por estudios más recientes (Fant 1984; Garrido y otros 1993; Prieto y otros 1994, 1995). Diversos estudios (Prieto y otros 1994, 1995; Llisterri y otros 1995) han señalado además que el desplazamiento del pico hacia la postónica puede presentar diferentes grados, en función 
de factores como la posición de la sílaba acentuada dentro de la palabra, la duración de la sílaba acentuada, la proximidad de ciertos límites prosódicos/sintácticos, factores rítmicos, o factores individuales, aunque todavía no se ha determinado muy claramente la contribución de cada uno. Este desplazamiento, en cambio, no parece darse si la función del acento es enfática, como señalan los datos presentados en De la Mota 1995. Por otro lado, algunos estudios han sugerido que puede haber también variación en el punto de inicio del movimiento ascendente, que la mayoría de descripciones sitúan al inicio de la sílaba tónica (Fant 1984; Garrido 1996), pero que según otros estudios puede retrasarse hasta la mitad de la sílaba tónica (Sosa 1999; Beckman y otros 2002; Face y Prieto 2007).

Esta variabilidad parece indicar que en español existe más de un patrón melódico para la expresión del acento. Hay dos patrones que aparecen recurrentemente en las diferentes descripciones:

1. Inicio del movimiento al comienzo de la tónica y final del mismo durante la tónica, preferentemente al final (Fant 1984; Garrido 1996, 2001). En el modelo autosegmental, este movimiento se ha asociado (Sosa 1999; Beckman y otros 2002; Face y Prieto 2007) con la configuración tonal $\mathrm{L}+\mathrm{H}^{*}$.

2. Inicio del movimiento en la tónica, preferentemente en la mitad, y final en la postónica. Es el movimiento que correspondería a la configuración tonal $\mathrm{L}^{*}+\mathrm{H}$ del modelo autosegmental (Sosa 1999; Beckman y otros 2002; Face y Prieto 2007).

Si bien la mayoría de los estudios señala que la tendencia mayoritaria en español es el uso de patrones con el final del movimiento ascendente en la postónica (Sosa 1995; D'Introno y otros 1995; Face 2000; Hualde 2000), Toledo 2000, 2001, 2003, 2004, en cambio, afirma que el mayoritario es el patrón con el pico en la tónica $\left(\mathrm{L}+\mathrm{H}^{*}\right)$. Tampoco está muy claro si la preferencia por uno u otro patrón se debe o no a factores lingüísticos, como la posición del acento en la palabra o la modalidad oracional, tal como sugieren Fernández y Martínez 2003.

Algunas propuestas, como Fernández y Martínez 2003 o Estebas y Prieto 2008, 2010 proponen inventarios de patrones más amplios, en los que se incluyen otros diferentes a los descritos anteriormente, como por ejemplo:

1. Pico en la pretónica al comienzo de la tónica, con descenso en la tónica $\left(\mathrm{H}+\mathrm{L}^{*}\right.$ y $\mathrm{H}^{*}+\mathrm{L}$ en el inventario de Fernández y Martínez 2003 y H+L* en Estebas y Prieto 2008, 2010).

2. Ascenso desde el inicio de la tónica hasta la postónica $\left(\mathrm{L}+>\mathrm{H}^{*}\right.$ en la propuesta de Estebas y Prieto 2008, 2010).

3. Nivel alto sostenido durante toda la tónica ( $\mathrm{H}^{*}$ en Estebas y Prieto 2008,2010 ). 
4. Nivel bajo sostenido durante toda la tónica ( $\mathrm{L}^{*}$ en Estebas y Prieto 2008,2010$)$.

Todo indica que la definición del inventario de patrones acentuales del español, así como de su frecuencia y usos, es aún una cuestión abierta, que a pesar de la intensa investigación de los últimos años requiere aún de nuevos estudios. No está muy claro cuál es el patrón acentual considerado «estándar», el inventario de patrones alternativos, ni los factores, lingüísticos o no, que determinan el uso de las diferentes variantes identificadas.

\section{Procedimiento}

\subsection{Corpus}

El material de habla analizado en este trabajo está formado por un conjunto de grupos acentuales extraído de cuatro corpus diferentes, grabado cada uno por un locutor profesional diferente, tres femeninos y uno masculino, todos ellos hablantes de español peninsular ${ }^{2}$. Solo en el caso de dos locutores (Mar y Ana) los enunciados eran los mismos; en los otros dos el número de enunciados y su contenido era diferente, pero todos ellos eran de habla leída y representativos de un estilo de habla neutro. En conjunto, los cuatro corpus suman 6.761 enunciados, con un total de 45.190 grupos acentuales, de los que 9.933 eran grupos iniciales y 23.797 grupos interiores, con lo que el total de grupos analizados ascendió a 33.730. La tabla 1 especifica el contenido de cada corpus.

\begin{tabular}{|l|c|c|c|}
\cline { 2 - 4 } \multicolumn{1}{c|}{} & $\begin{array}{c}\text { Grupos acentuales } \\
\text { iniciales }\end{array}$ & $\begin{array}{c}\text { Grupos acentuales } \\
\text { interiores }\end{array}$ & Total \\
\hline Mar & 3.542 & 8.471 & 12.013 \\
\hline Jordi & 2.092 & 5.489 & 7.581 \\
\hline Ana & 3.638 & 8.345 & 11.983 \\
\hline Esm & 661 & 1.492 & 2.153 \\
\hline Total & 9.933 & 23.797 & 33.730 \\
\hline
\end{tabular}

TABLA 1: Número de grupos acentuales iniciales e interiores del corpus analizado

${ }^{2}$ Mi agradecimiento a Barcelona Media y Telefónica Investigación y Desarrollo, propietarias de los corpus, por permitir su utilización para la realización de este trabajo. 
Para cada uno de los enunciados de los cuatro corpus se dispone de la transcripción ortográfica y fonética asociada con la señal de habla, en ficheros TextGrid de Praat (programa para el análisis del habla descrito en Boersma 2001), que se utilizaron también como material de partida para el análisis que se describe en el apartado 3. Tanto la transcripción fonética como el alineamiento con la señal de habla se han obtenido con herramientas automáticas y en la mayor parte de los enunciados no se han corregido manualmente. También se ha obtenido de manera automática, a partir de esta segmentación fonética, la segmentación de los enunciados en sílabas, grupos acentuales, grupos fónicos y grupos entonativos. Se ha comprobado que el resultado obtenido es de una calidad suficiente como para no desvirtuar los análisis realizados a partir de este material.

\subsection{Metodología de extracción de patrones}

Para la obtención automática del inventario de patrones melódicos descrito en este trabajo, todo este material fue procesado utilizando la herramienta automática de estilización, anotación y modelización de contornos descrita en Garrido 2010. Esta herramienta toma como punto de partida la segmentación en unidades fonéticas de los enunciados descrita anteriormente, y tras estilizar y anotar los contornos, realiza un recuento de los diferentes patrones melódicos contenidos en el corpus de entrada. Está compuesta por una serie de scripts de Praat y $R$ (R Development Core Team 2008), y ha sido desarrollada íntegramente por el autor, aplicando los principios del modelo de representación y modelización de contornos propuesto en Garrido 1996, 2001.

La tarea de estilización se lleva a cabo mediante un script de Praat que llama al algoritmo incluido en Praat para este fin, que estiliza las curvas melódicas buscando puntos de inflexión relevantes desde el punto de vista acústico (cambios en la pendiente que sobrepasan un determinado umbral previamente determinado). Una descripción más detallada del proceso de estilización puede encontrarse en el manual de Praat. Este umbral puede ser configurado por el usuario y durante el proceso de desarrollo del sistema fue convenientemente ajustado a 1 semitono para obtener representaciones lo más estilizadas posible (con la consiguiente eliminación de variaciones micromelódicas y segmentos sordos), pero manteniendo al máximo la equivalencia perceptiva con el original, tal como se pudo comprobar en el experimento de percepción descrito en Garrido 2010. El objetivo es, en suma, obtener close-copy stylizations, al estilo de la aproximación IPO (T'Hart y otros 1990), pero de forma automática en lugar de manual. Esta aproximación, al basarse en criterios acústicos, puede provocar que en algunos casos se mantengan en 
la curva estilizada algunos puntos que corresponderían a variaciones micromelódicas muy pronunciadas, pero se trata, según nuestra experiencia, de casos poco significativos, que por otro lado no afectan a la equivalencia perceptiva de los contornos estilizados obtenidos. Los valores de F0 correspondientes a los puntos de inflexión que componen la curva estilizada resultante se anotan, alineados temporalmente con la señal de habla, en un tier del TextGrid de salida, tal como puede observarse en el ejemplo de la figura 1.

Para el proceso de anotación se emplea un conjunto de scripts de Praat y R que asignan los puntos de inflexión retenidos en la estilización a un nivel «Pico» $(\mathrm{P}) \mathrm{o}$ «Valle» $(\mathrm{V})$, según se encuentre en la parte alta o baja del rango tonal del grupo fónico que los contiene. La asignación de cada punto a un nivel u otro se lleva a cabo a partir del cálculo de una recta de regresión que define el «tono medio» a lo largo de cada grupo fónico: si el punto está por encima de esa línea media, se asigna al nivel P; si está por debajo, se asigna al V. Después, en una nueva iteración del proceso, aquellos puntos de inflexión que están claramente por encima de nivel medio calculado para $\mathrm{P}$ y por debajo del nivel medio de $\mathrm{V}$ cambian su etiqueta $\mathrm{P}$ o $\mathrm{V}$ ya asignada por otra etiqueta diferente $(\mathrm{P}+\mathrm{y} \mathrm{V}-$, respectivamente). Finalmente, una última iteración del proceso elimina de la anotación los puntos de inflexión con

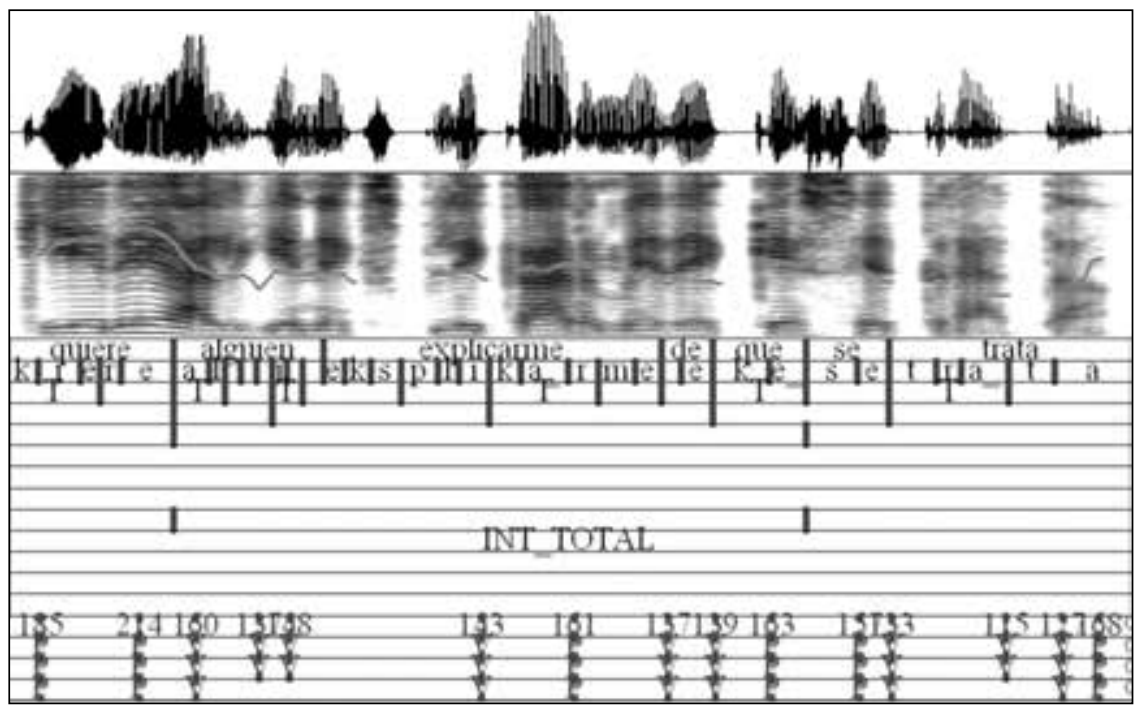

Figura 1: Ejemplo de TextGrid de Praat resultante del proceso de estilización y anotación del enunciado ¿Q Quiere alguien explicarme de qué se trata? pronunciado por la locutora Esm 
etiquetas consideradas redundantes, dado que pueden inferirse de la etiqueta de los puntos anterior y posterior. Así, se eliminará un punto de inflexión etiquetado como $\mathrm{P}$ si los puntos anterior y posterior también tienen la misma etiqueta, porque se considera que el nivel tonal del mismo es una mera interpolación del que le sigue y del que le precede. La salida de cada una de estas iteraciones se anota, alineada siempre en el tiempo con la señal de habla, en tres nuevos tiers que se añaden al TextGrid de salida, tal como puede observarse en la figura 1.

Tras la estilización y anotación, otro script de Praat se encarga de extraer automáticamente de los TextGrid anotados los patrones melódicos que componen cada enunciado de entrada. Se entiende aquí por patrón melódico, siguiendo el modelo de descripción de los contornos melódicos propuesto en Garrido 1996, 2001, una serie de puntos de inflexión anotados que se da en el ámbito de un GA. Cada patrón se identifica por la cadena de puntos de inflexión que lo componen, representados por la etiqueta que representa su altura tonal (V-, V, P, o P+), más una serie de letras y números que representan la sílaba del GA en la que aparece (0 para la sílaba tónica del grupo, 1 para la postónica, -1 para la pretónica, etc.), y su posición con respecto al núcleo silábico de la misma (I si está cerca del inicio; M si está cerca del centro; F si está cerca del final). Si el GA no contiene ningún punto de inflexión, se eti-

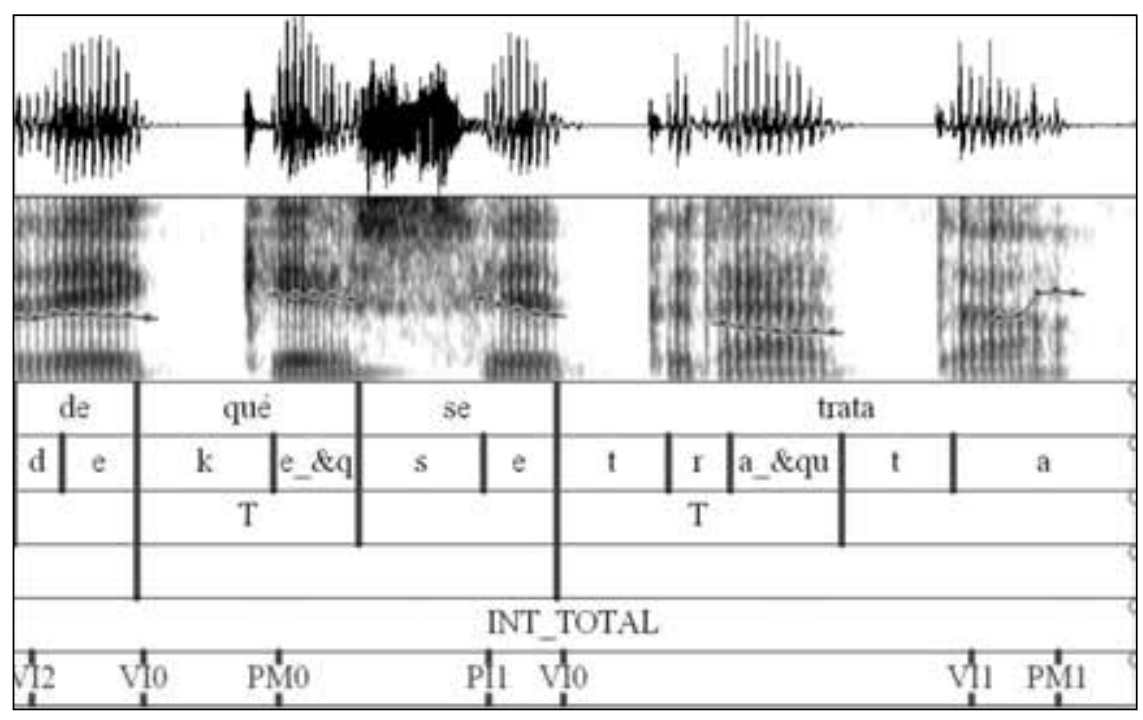

FIgURA 2: Ejemplo de notación de los dos últimos patrones melódicos del enunciado ¿Quiere alguien explicarme de qué se trata? pronunciado por la locutora Esm 
queta como 0. Así, un patrón entonativo etiquetado como VI0_VI1_PM1 (figura 2) está formado por tres puntos de inflexión: el primero está anotado como V, y se sitúa en los alrededores del inicio del núcleo silábico de la sílaba tónica; el segundo tiene también la etiqueta $\mathrm{V}$ y se sitúa cerca del inicio del núcleo silábico de la sílaba postónica (1), y el tercero tiene la etiqueta $\mathrm{P}$ y se sitúa cerca del centro del núcleo silábico de la misma postónica.

La salida de esta herramienta es un listado de los diferentes patrones identificados, organizados en función del tipo de GA (final de grupo entonativo no final de oración y final de oración), el número de sílabas del grupo, la posición de la sílaba tónica dentro del grupo (normalmente la primera, pero en determinados grupos iniciales puede haber una o más átonas antes de la tónica, según la definición establecida anteriormente) y la modalidad oracional del enunciado (enunciativa, interrogativa y exclamativa), con el número de apariciones de cada uno en el corpus analizado. Este listado es el que ha utilizado como material de base para el análisis que aquí se presenta.

\section{Resultados}

El listado total de patrones obtenidos es demasiado grande como para abordar un análisis global de los mismos, por lo que se ha optado por hacer una selección de los grupos acentuales más frecuentes (los de una, dos y tres sílabas), y limitar los datos presentados aquí a los patrones más frecuentes que se dan en estos grupos. Así, en los siguientes apartados se presenta una serie de tablas con un listado de los patrones más frecuentes para cada una de las categorías consideradas: patrones iniciales y patrones interiores, que son los relacionados con el acento.

\subsection{Patrones iniciales}

Las tablas 2-4 muestran los patrones más frecuentes en los corpus analizados para los GA iniciales de 1, 2 y 3 sílabas con acento en la primera sílaba; las tablas 5-6, los patrones más frecuentes en los GA con acento en la segunda sílaba (GA de 2 y 3 sílabas); y finalmente, la tabla 7 presenta los patrones más frecuentes en los GA de 3 sílabas con acento en la tercera sílaba. 


\begin{tabular}{|l|c|c|c|c|}
\hline \multicolumn{1}{|c|}{ Patrón } & $\begin{array}{c}\text { Número } \\
\text { sílabas GA }\end{array}$ & $\begin{array}{c}\text { Sílaba } \\
\text { acentuada GA }\end{array}$ & $\begin{array}{c}\text { Modalidad } \\
\text { oracional }\end{array}$ & $\begin{array}{c}\text { Número } \\
\text { apariciones }\end{array}$ \\
\hline PI0 & 1 & 1 & AFIRM & 98 \\
\hline VM0 & 1 & 1 & AFIRM & 83 \\
\hline VI0_PM0 & 1 & 1 & AFIRM & 71 \\
\hline VI0 & 1 & 1 & AFIRM & 64 \\
\hline PI0 & 1 & 1 & INT & 60 \\
\hline PM0 & 1 & 1 & AFIRM & 57 \\
\hline VI0_PF0 & 1 & 1 & AFIRM & 38 \\
\hline VI0 & 1 & 1 & INT & 25 \\
\hline PI0_PM0 & 1 & 1 & AFIRM & 24 \\
\hline VI0_VF0 & & 1 & AFIRM & 15 \\
\hline
\end{tabular}

TABLA 2: Patrones iniciales más frecuentes en los GA de una sílaba

\begin{tabular}{|l|c|c|c|c|}
\hline Patrón & $\begin{array}{c}\text { Número } \\
\text { sílabas GA }\end{array}$ & $\begin{array}{c}\text { Sílaba } \\
\text { acentuada GA }\end{array}$ & $\begin{array}{c}\text { Modalidad } \\
\text { oracional }\end{array}$ & $\begin{array}{c}\text { Número } \\
\text { apariciones }\end{array}$ \\
\hline PI0 & 2 & 1 & AFIRM & 59 \\
\hline VI0_PI1 & 2 & 1 & AFIRM & 58 \\
\hline VM0_PI1 & 2 & 1 & AFIRM & 46 \\
\hline PI0_PI1 & 2 & 1 & AFIRM & 45 \\
\hline VI0_PM0 & 2 & 1 & AFIRM & 33 \\
\hline VI0_PM1 & 2 & 1 & AFIRM & 31 \\
\hline VM0 & 2 & 1 & AFIRM & 27 \\
\hline VI0 & 2 & 1 & AFIRM & 26 \\
\hline PM0 & 2 & 1 & AFIRM & 26 \\
\hline VI0_PF0 & & 1 & AFIRM & 23 \\
\hline
\end{tabular}

TABLA 3: Patrones iniciales más frecuentes en los GA de dos sílabas con acento en la primera sílaba 


\begin{tabular}{|l|c|c|c|c|}
\hline \multicolumn{1}{|c|}{ Patrón } & $\begin{array}{c}\text { Número } \\
\text { sílabas GA }\end{array}$ & $\begin{array}{c}\text { Sílaba } \\
\text { acentuada GA }\end{array}$ & $\begin{array}{c}\text { Modalidad } \\
\text { oracional }\end{array}$ & $\begin{array}{c}\text { Número } \\
\text { apariciones }\end{array}$ \\
\hline VI0_PI1 & 3 & 1 & AFIRM & 31 \\
\hline PI0_PI1 & 3 & 1 & AFIRM & 26 \\
\hline VI0_PM1 & 3 & 1 & AFIRM & 19 \\
\hline VI0_PM0 & 3 & 1 & AFIRM & 19 \\
\hline VI0_PI1_VF2 & 3 & 1 & AFIRM & 19 \\
\hline VM0_PI1 & 3 & 1 & AFIRM & 15 \\
\hline PI0_PM1 & 3 & 1 & AFIRM & 15 \\
\hline PI0_PI2 & 3 & 1 & AFIRM & 11 \\
\hline PI0_PI1_VI2 & 3 & 1 & AFIRM & 10 \\
\hline PI0_PI1_VM2 & 3 & & & 11 \\
\hline
\end{tabular}

TABLA 4: Patrones iniciales más frecuentes en los GA de tres sílabas con acento en la primera sílaba

\begin{tabular}{|l|c|c|c|c|}
\hline \multicolumn{1}{|c|}{ Patrón } & $\begin{array}{c}\text { Número } \\
\text { sílabas GA }\end{array}$ & $\begin{array}{c}\text { Sílaba } \\
\text { acentuada GA }\end{array}$ & $\begin{array}{c}\text { Modalidad } \\
\text { oracional }\end{array}$ & $\begin{array}{c}\text { Número } \\
\text { apariciones }\end{array}$ \\
\hline VI-1_VI0_PM0 & 2 & 2 & AFIRM & 44 \\
\hline PI-1_VI0_PM0 & 2 & 2 & AFIRM & 16 \\
\hline VI-1_VI0_PM0 & 2 & 2 & INT & 15 \\
\hline VM-1_VI0_PM0 & 2 & 2 & AFIRM & 13 \\
\hline VM-1_VI0_PM0 & 2 & 2 & INT & 13 \\
\hline VI-1_VI0_PF0 & 2 & 2 & AFIRM & 13 \\
\hline VI-1 & 2 & 2 & AFIRM & 13 \\
\hline VI-1_VF-1_PM0 & 2 & 2 & INT & 10 \\
\hline VM-1 & 2 & 2 & AFIRM & 9 \\
\hline PI-1_VI0_PM0 & 2 & 2 & INT & 9 \\
\hline
\end{tabular}

TABLA 5: Patrones iniciales más frecuentes en los GA de dos sílabas con acento en la segunda sílaba 


\begin{tabular}{|l|c|c|c|c|}
\hline \multicolumn{1}{|c|}{ Patrón } & $\begin{array}{c}\text { Número } \\
\text { sílabas GA }\end{array}$ & $\begin{array}{c}\text { Sílaba } \\
\text { acentuada GA }\end{array}$ & $\begin{array}{c}\text { Modalidad } \\
\text { oracional }\end{array}$ & $\begin{array}{c}\text { Número } \\
\text { apariciones }\end{array}$ \\
\hline VI-1_VI0_PM0 & 3 & 2 & AFIRM & 58 \\
\hline VI-1_VI0_PM0_PI1 & 3 & 2 & AFIRM & 27 \\
\hline VI-1_VI0_PF0 & 3 & 2 & AFIRM & 25 \\
\hline VI-1_VF-1_PM0 & 3 & 2 & AFIRM & 20 \\
\hline VM-1_VI0_PM0 & 3 & 2 & AFIRM & 17 \\
\hline PI-1_VI0_PM0 & 3 & 2 & AFIRM & 16 \\
\hline VI-1_VI0_PM0_PM1 & 3 & 2 & AFIRM & 15 \\
\hline VM-1_VI0_PI1 & 3 & 2 & AFIRM & 14 \\
\hline VI-1_VI0_PI1 & 3 & AFIRM & 14 \\
\hline VI-1_VF-1_PI1 & 3 & AFIRM & 11 \\
\hline
\end{tabular}

TABLA 6: Patrones iniciales más frecuentes en los GA de tres sílabas con acento en la segunda sílaba

\begin{tabular}{|l|c|c|c|c|}
\hline \multicolumn{1}{|c|}{ Patrón } & $\begin{array}{c}\text { Número } \\
\text { sílabas GA }\end{array}$ & $\begin{array}{c}\text { Sílaba } \\
\text { acentuada GA }\end{array}$ & $\begin{array}{c}\text { Modalidad } \\
\text { oracional }\end{array}$ & $\begin{array}{c}\text { Número } \\
\text { apariciones }\end{array}$ \\
\hline VI-2_VI0_PM0 & 3 & 3 & AFIRM & 14 \\
\hline VM-2_VI0_PM0 & 3 & 3 & AFIRM & 8 \\
\hline VI-2_VI0_PF0 & 3 & 3 & AFIRM & 7 \\
\hline VI-2_VI-1_PM0 & 3 & 3 & AFIRM & 6 \\
\hline VM-2_VI0_PF0 & 3 & 3 & AFIRM & 4 \\
\hline PI-2_VI-1_VI0_PM0 & 3 & 3 & AFIRM & 4 \\
\hline VM-2_VI-1_PF0 & 3 & 3 & AFIRM & 3 \\
\hline VI-2_VI0_PM0_PF0 & 3 & 3 & AFIRM & 3 \\
\hline VI-2_VI0 & 3 & 3 & AFIRM & 3 \\
\hline VI-2_VF-1_PI0 & 3 & 3 & AFIRM & 3 \\
\hline
\end{tabular}

TABLA 7: Patrones iniciales más frecuentes en los GA de tres sílabas con acento en la tercera sílaba 
El análisis de las tablas 2-4 revela, en primer lugar, que si la sílaba tónica es la primera del GA, el patrón más frecuente es PI0, es decir, un único punto de inflexión en el nivel alto al inicio de la sílaba tónica. Es el patrón más frecuente en los GA de una sola sílaba, y también en los GA de dos sílabas. La figura 3 presenta un ejemplo de este patrón.

También es frecuente, dentro de los grupos de dos sílabas, PI0_PI1, es decir, una inflexión en nivel alto al inicio de la tónica y otra, también de nivel $\mathrm{P}$, al inicio de la postónica (es el más frecuente en el caso de las interrogativas de dos sílabas). La figura 4 muestra un ejemplo de este patrón.

Son igualmente frecuentes, aunque algo menos que los anteriores, patrones con una inflexión inicial $\mathrm{V}$ y otra $\mathrm{P}$, que puede aparecer en la mitad de la sílaba tónica (VI0_PM0) o en el inicio de la postónica (VI0_PI1, VM0_PI1), si el grupo tiene más de una sílaba. Las figuras 5 y 6 ilustran ambos tipos de patrones.

También aparecen entre los más frecuentes patrones con una sola inflexión de nivel V (VI0, VM0). La figura 7 ilustra este caso.

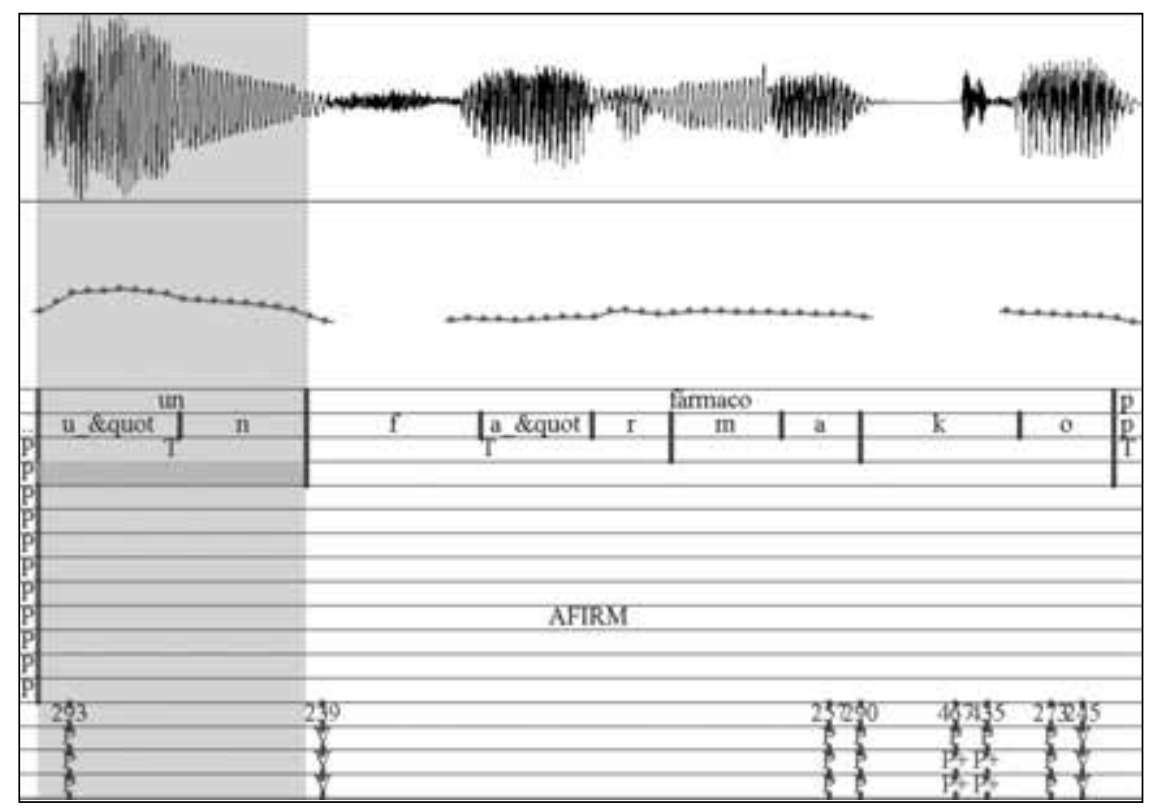

Figura 3: Ejemplo de patrón inicial PI0 en el grupo acentual un, dentro del enunciado Un fármaco poco usado por sus riesgos pronunciado por la locutora Ana. Las etiquetas correspondientes a los puntos de inflexión del patrón aparecen en la zona sombreada del último tier, alineadas en el tiempo con la curva original de la parte superior 


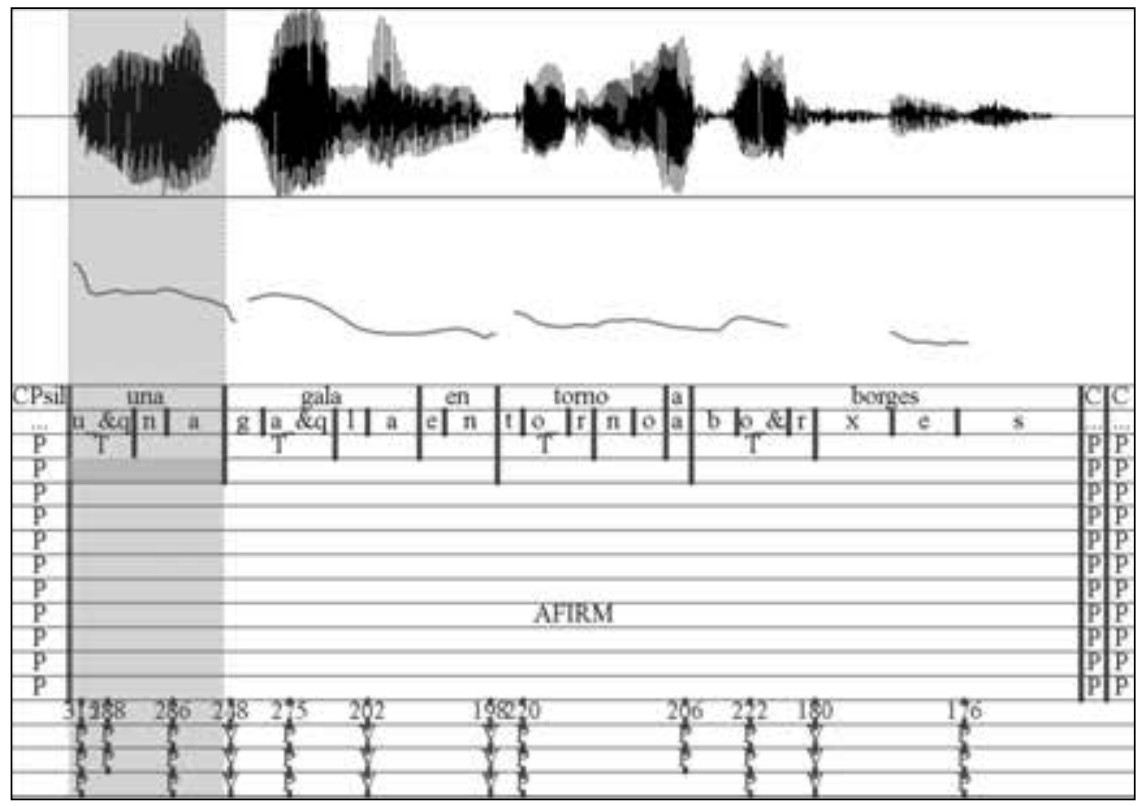

FIgURA 4: Ejemplo de patrón inicial PI0_PI1 en el grupo acentual una, dentro del enunciado Una gala en torno a Borges pronunciado por la locutora Mar. Las etiquetas correspondientes a los puntos de inflexión del patrón aparecen en la zona sombreada del último tier, alineadas en el tiempo con la curva original de la parte superior

Si la sílaba tónica es la segunda del grupo (tablas 5 y 6), el patrón más frecuente, con independencia del número de sílabas del GA, es VI-1_VI0_PM0, es decir, con una inflexión de nivel V al inicio y otra de nivel $\mathrm{P}$ en la mitad de la sílaba tónica. Con menor frecuencia, el pico puede darse también al final de la sílaba tónica (VI-1_VI0_PF0, en los GA de dos y tres sílabas), en la postónica (VI-1_VI0_PM1) o al inicio de la tónica (VI-1_VF-1_PI0). Las figuras 8-10 ilustran estos patrones.

Finalmente, si la tónica es la tercera (tabla 7), el patrón más frecuente es VI-2_VI0_PM0, seguido de VM-2_VI0_PM0. En ambos casos, el inicio del ascenso se da al comienzo de la sílaba tónica, y el pico se sitúa en la mitad de la sílaba tónica. Con menor frecuencia, también puede darse al final de la sílaba tónica (VI-2_VI0_PF0, VM-2_VI0_PF0). Las figuras 11 y 12 ilustran estos dos tipos de patrones. 


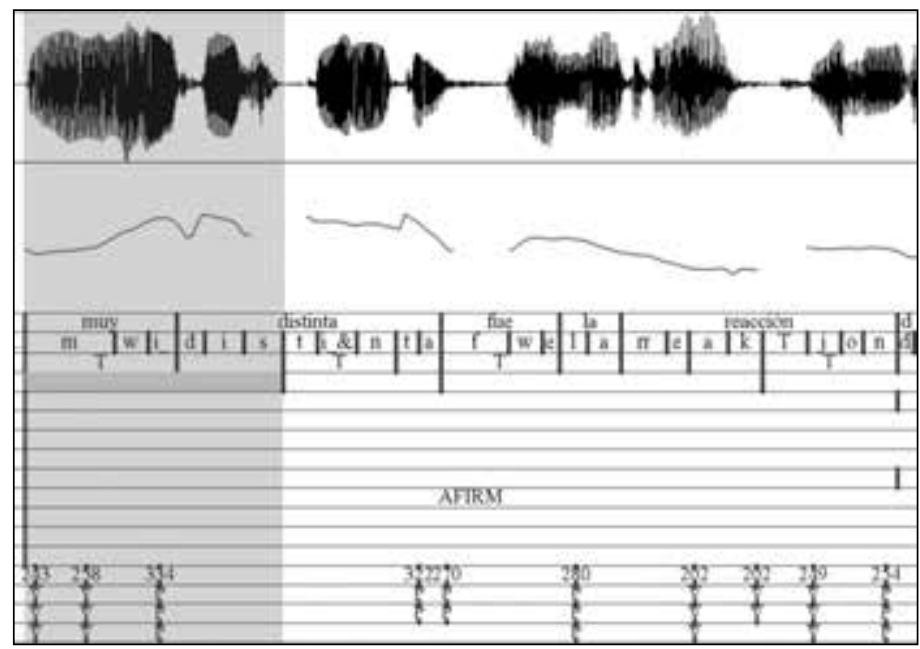

FIGURA 5: Ejemplo de patrón inicial VI0_PM0 en el grupo acentual muy dis, dentro del enunciado Muy distinta fue la reacción de la ex primera ministra británica, Margaret Tatcher pronunciado por la locutora Mar. Las etiquetas correspondientes a los puntos de inflexión del patrón aparecen en la zona sombreada del último tier, alineadas en el tiempo con la curva original de la parte superior

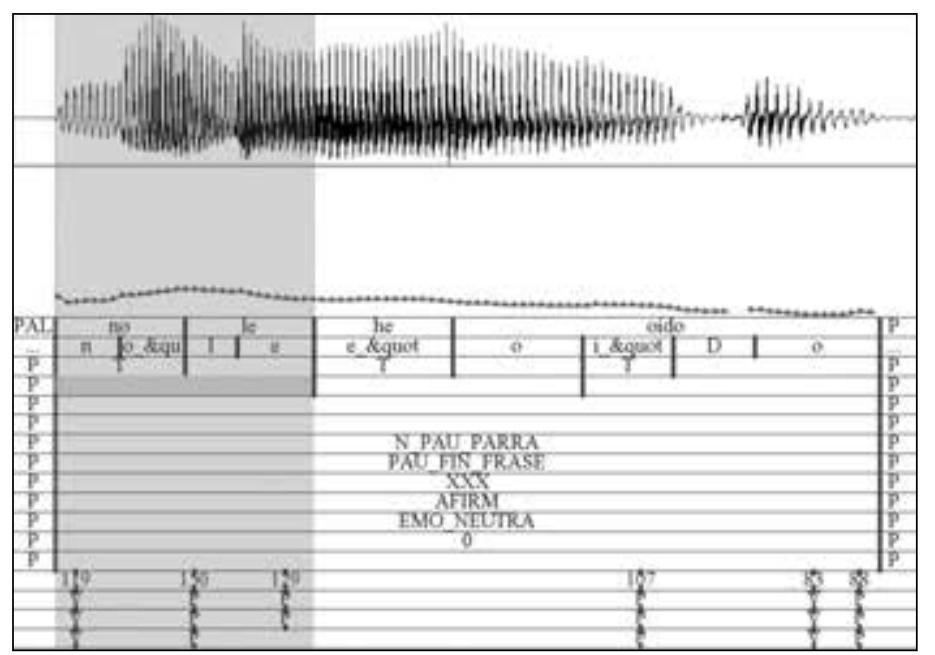

FIGURA 6: Ejemplo de patrón inicial VI0_PI1 en el grupo acentual no le, dentro del enunciado No le he oído pronunciado por el locutor Jordi. Las etiquetas correspondientes a los puntos de inflexión del patrón aparecen en la zona sombreada del último tier, alineadas en el tiempo con la curva original de la parte superior 


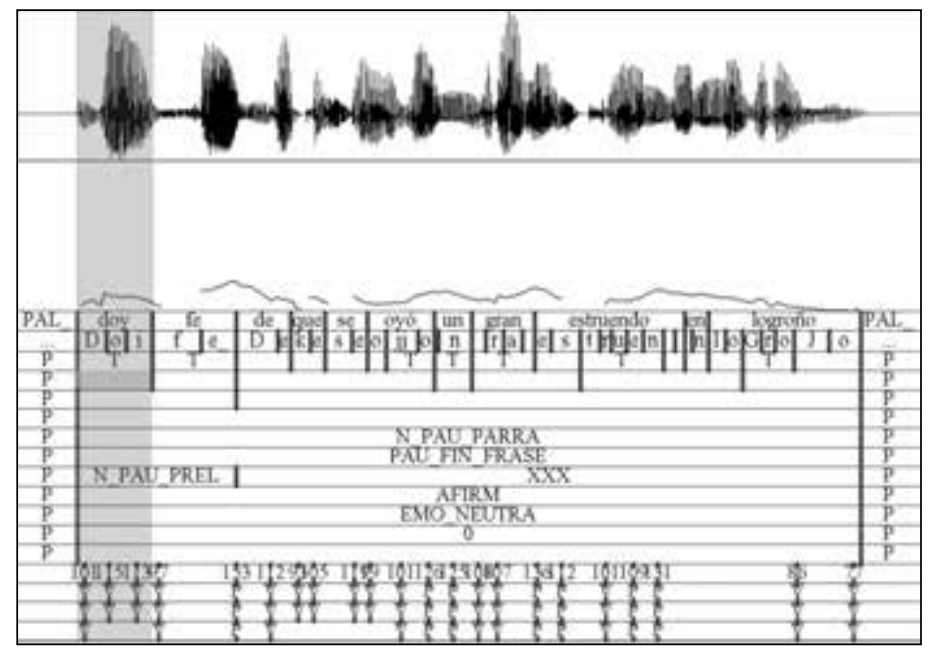

Figura 7: Ejemplo de patrón inicial VI0 en el grupo acentual doy, dentro del enunciado Doy fe de que se oyó un gran estruendo en Logroño pronunciado por el locutor Jordi. Las etiquetas correspondientes a los puntos de inflexión del patrón aparecen en la zona sombreada del último tier, alineadas en el tiempo con la curva original de la parte superior

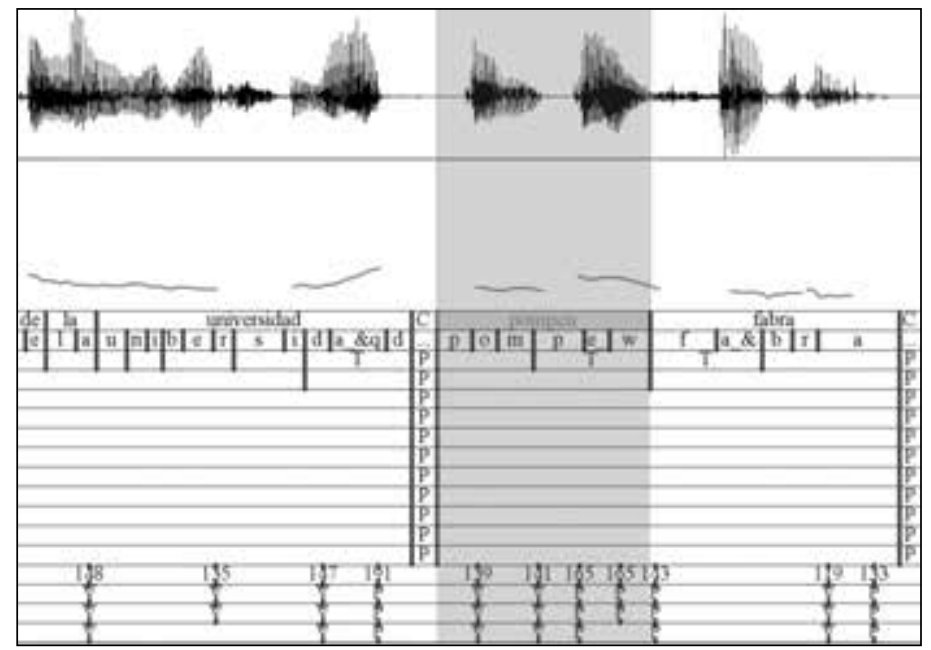

FIGURA 8: Ejemplo de patrón inicial VI-1_VI0_PM0 en el grupo acentual pompeu, dentro del enunciado En el departamento de Sociales de la Universidad Pompeu Fabra pronunciado por el locutor Esm. Las etiquetas correspondientes a los puntos de inflexión del patrón aparecen en la zona sombreada del último tier, alineadas en el tiempo con la curva original de la parte superior 


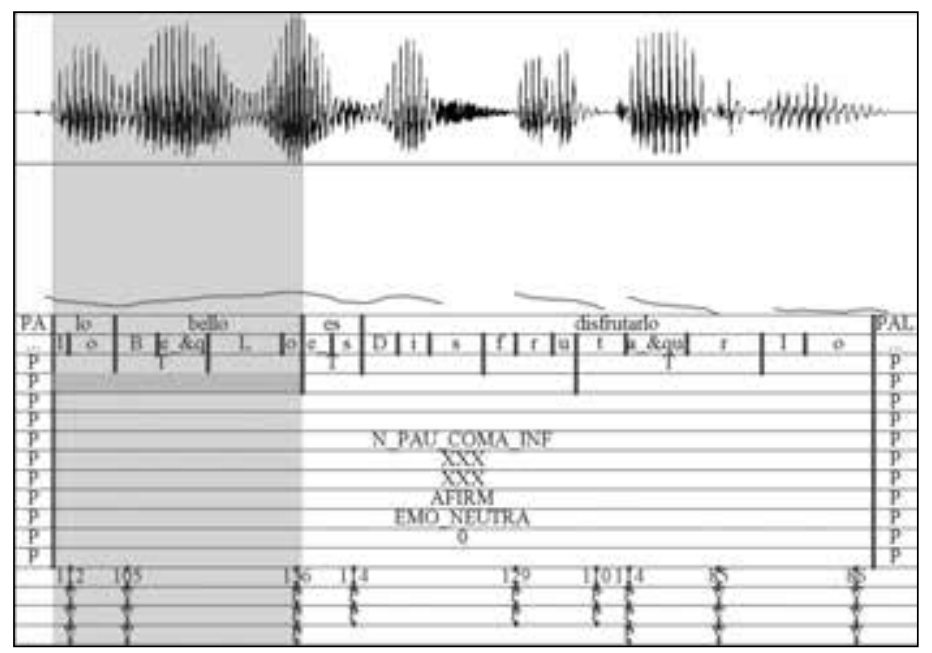

FIGURA 9: Ejemplo de patrón inicial VI-1_VI0_PM1 en el grupo acentual lo bello, dentro del enunciado Lo bello es disfrutarlo, no agarrarse del chongo por tales detalles pronunciado por el locutor Jordi. Las etiquetas correspondientes a los puntos de inflexión del patrón aparecen en la zona sombreada del último tier, alineadas en el tiempo con la curva original de la parte superior

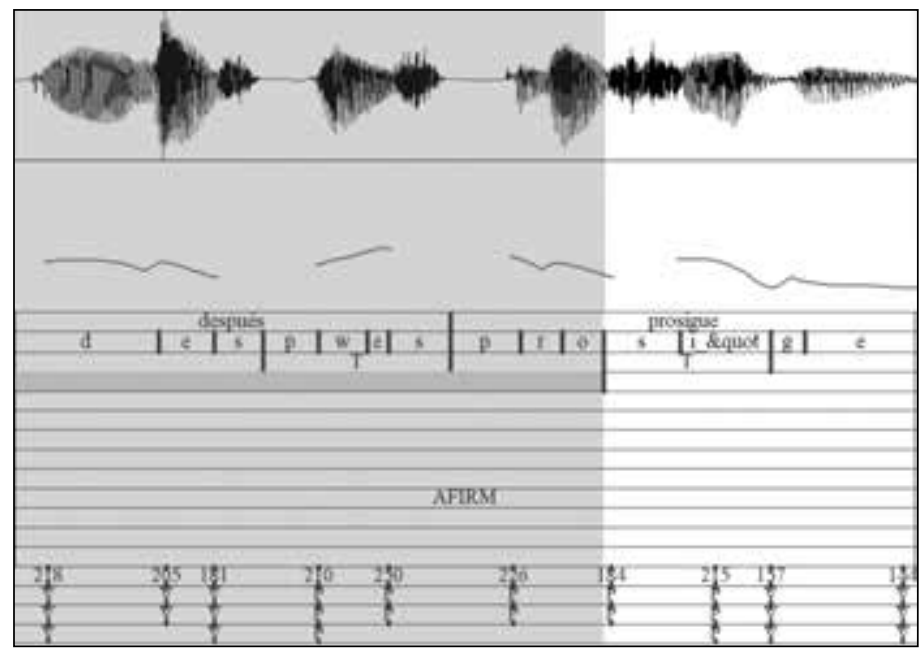

FIGURA 10: Ejemplo de patrón inicial VI-1_VF-1_PI0 en el grupo acentual después pro, dentro del enunciado Después prosigue: ahora estás en el Bronx, en Nueva York pronunciado por la locutora Ana. Las etiquetas correspondientes a los puntos de inflexión del patrón aparecen en la zona sombreada del último tier, alineadas en el tiempo con la curva original de la parte superior 


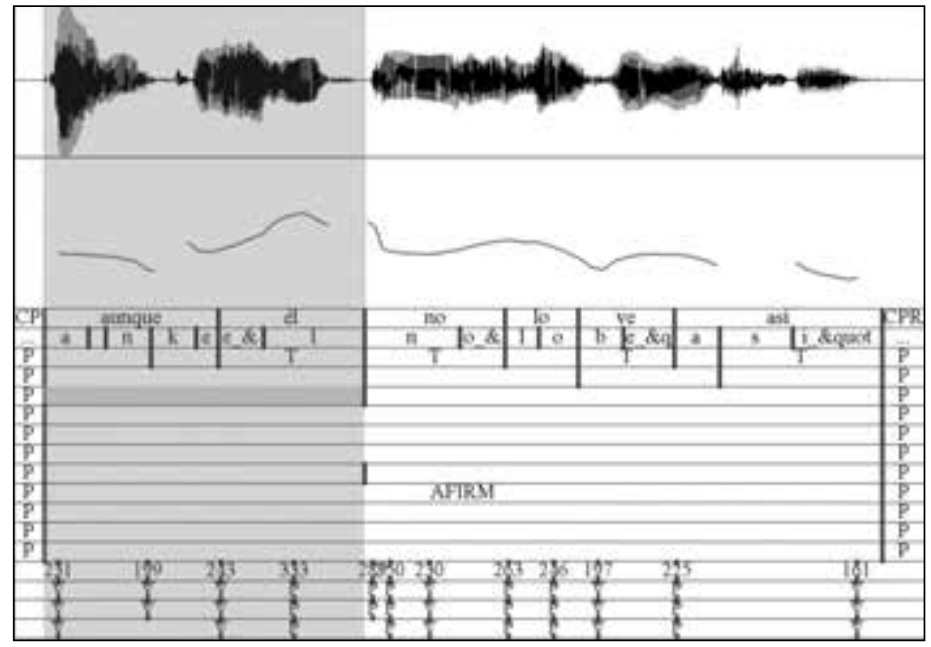

FigURA 11: Ejemplo de patrón inicial VI-2_VI0_PM0 en el grupo acentual aunque él, dentro del enunciado Aunque él no lo ve así pronunciado por la locutora Mar. Las etiquetas correspondientes a los puntos de inflexión del patrón aparecen en la zona sombreada del último tier, alineadas en el tiempo con la curva original de la parte superior

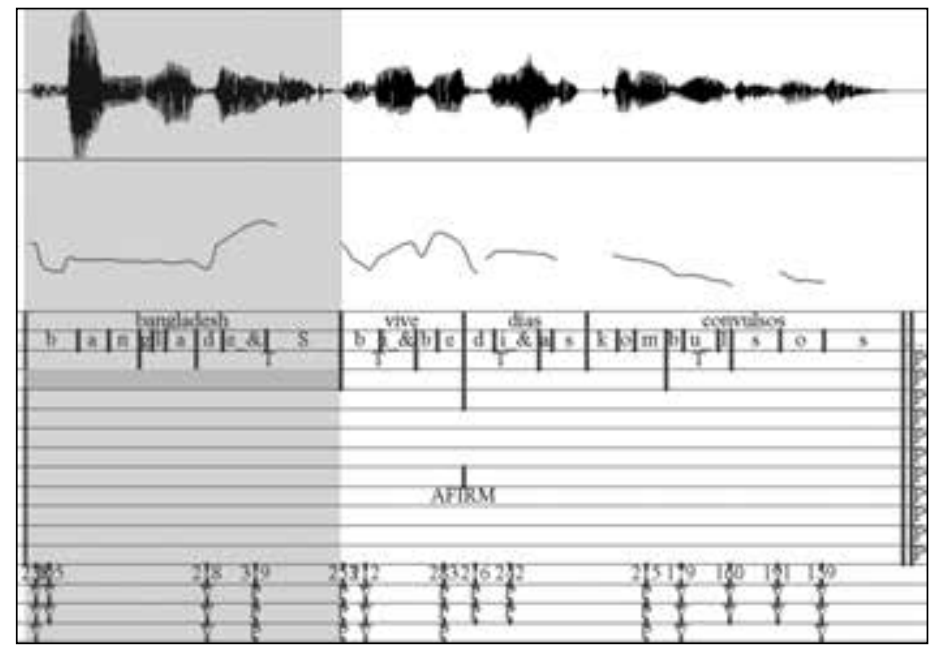

FIGURA 12: Ejemplo de patrón inicial VI-2_VI0_PF0 en el grupo acentual bangla desh, dentro del enunciado Bangla Desh vive días convulsos pronunciado por la locutora Mar. Las etiquetas correspondientes a los puntos de inflexión del patrón aparecen en la zona sombreada del último tier, alineadas en el tiempo con la curva original de la parte superior 


\subsection{Patrones interiores}

Las tablas 8-10 muestran los patrones más frecuentes en los corpus analizados para los grupos acentuales interiores de 1, 2 y 3 sílabas, respectivamente.

\begin{tabular}{|l|c|c|c|c|}
\hline \multicolumn{1}{|c|}{ Patrón } & $\begin{array}{c}\text { Número } \\
\text { sílabas GA }\end{array}$ & $\begin{array}{c}\text { Sílaba } \\
\text { acentuada GA }\end{array}$ & $\begin{array}{c}\text { Modalidad } \\
\text { oracional }\end{array}$ & $\begin{array}{c}\text { Número } \\
\text { apariciones }\end{array}$ \\
\hline 0 & 1 & 1 & AFIRM & 598 \\
\hline VI0_PM0 & 1 & 1 & AFIRM & 320 \\
\hline PM0 & 1 & 1 & AFIRM & 193 \\
\hline PI0 & 1 & 1 & AFIRM & 191 \\
\hline VI0 & 1 & 1 & AFIRM & 171 \\
\hline VF0 & 1 & 1 & AFIRM & 147 \\
\hline VI0_PF0 & 1 & 1 & AFIRM & 135 \\
\hline 0 & 1 & 1 & INT & 102 \\
\hline PF0 & 1 & 1 & AFIRM & 94 \\
\hline VM0 & 1 & 1 & AFIRM & 52 \\
\hline
\end{tabular}

TABLA 8: Patrones interiores más frecuentes en los GA de una sílaba

\begin{tabular}{|l|c|c|c|c|}
\hline \multicolumn{1}{|c|}{ Patrón } & $\begin{array}{c}\text { Número } \\
\text { sílabas GA }\end{array}$ & $\begin{array}{c}\text { Sílaba } \\
\text { acentuada GA }\end{array}$ & $\begin{array}{c}\text { Modalidad } \\
\text { oracional }\end{array}$ & $\begin{array}{c}\text { Número } \\
\text { apariciones }\end{array}$ \\
\hline 0 & 2 & 1 & AFIRM & 529 \\
\hline VI0_PM0 & 2 & 1 & AFIRM & 351 \\
\hline PI1 & 2 & 1 & AFIRM & 283 \\
\hline VI0_PI1 & 2 & 1 & AFIRM & 228 \\
\hline PM0 & 2 & 1 & AFIRM & 174 \\
\hline PI0 & 2 & 1 & AFIRM & 168 \\
\hline VI0_PM0_PI1 & 2 & 1 & AFIRM & 150 \\
\hline 0 & 2 & 1 & INT & 147 \\
\hline VF1 & 2 & 1 & AFIRM & 136 \\
\hline VI0 & 2 & 1 & AFIRM & 130 \\
\hline
\end{tabular}

TABLA 9: Patrones interiores más frecuentes en los GA de dos sílabas 


\begin{tabular}{|l|c|c|c|c|}
\hline \multicolumn{1}{|c|}{ Patrón } & $\begin{array}{c}\text { Número } \\
\text { sílabas GA }\end{array}$ & $\begin{array}{c}\text { Sílaba } \\
\text { acentuada GA }\end{array}$ & $\begin{array}{c}\text { Modalidad } \\
\text { oracional }\end{array}$ & $\begin{array}{c}\text { Número } \\
\text { apariciones }\end{array}$ \\
\hline 0 & 3 & 1 & AFIRM & 328 \\
\hline VI0_PM0 & 3 & 1 & AFIRM & 210 \\
\hline PI1 & 3 & 1 & AFIRM & 162 \\
\hline VI0_PI1 & 3 & 1 & AFIRM & 120 \\
\hline VI0 & 3 & 1 & AFIRM & 120 \\
\hline VF2 & 3 & 1 & AFIRM & 114 \\
\hline 0 & 3 & 1 & INT & 88 \\
\hline VI0_PM0_PI2 & 3 & 1 & AFIRM & 79 \\
\hline PI1_VI2 & 3 & 1 & AFIRM & 73 \\
\hline PI0 & 3 & 1 & AFIRM & 72 \\
\hline
\end{tabular}

TABLA 10: Patrones interiores más frecuentes en los GA de tres sílabas

Lo primero que destaca de la observación de las tablas es la predominancia de los grupos con ausencia de inflexión (0), que son los más frecuentes en los tres grupos (uno, dos y tres sílabas), y que se darían, de acuerdo con los ejemplos de las figuras 13 y 14, en grupos de transición entre dos patrones que acaban y empiezan con nivel P.

Después de 0, el patrón más frecuente es VI0_PM0 (inicio del ascenso del pico al comienzo de la sílaba tónica, y pico en las proximidades del centro del núcleo silábico), con independencia del número de sílabas del GA (figuras 15 y 16 ).

Tras este, se sitúan PM0 (una única inflexión en la mitad de la sílaba tónica) y PI0 (inflexión al inicio de la sílaba tónica), en los grupos de una sílaba, y PI1 y VI0_PI1, en los de dos y tres sílabas. Las figuras 17 y 18 muestran ejemplos de PI0 y PI1. En ambos ejemplos se observa que la inflexión que marca el inicio del ascenso tonal en estos casos se sitúa poco antes del inicio del GA del patrón, aún en la sílaba pretónica. 


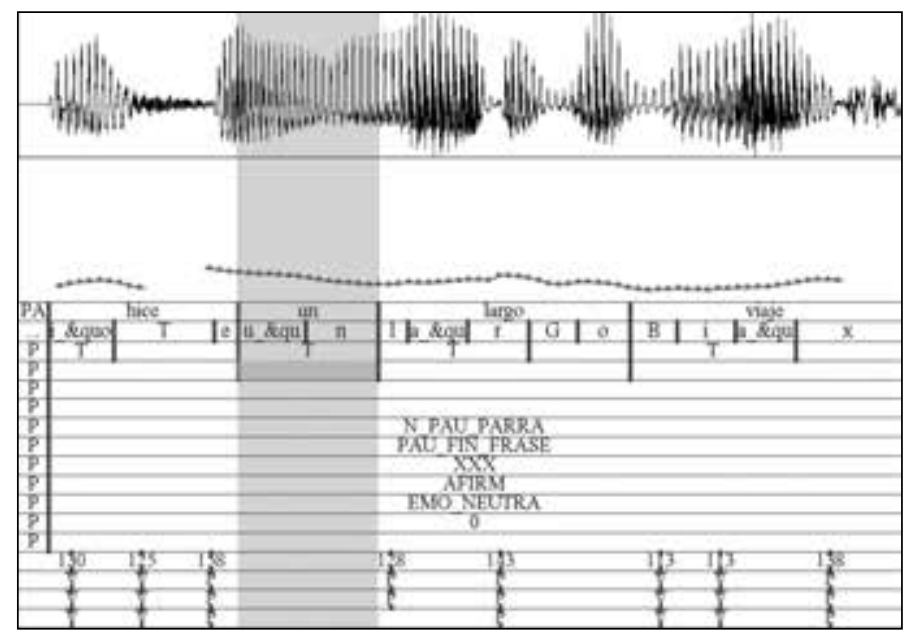

Figura 13: Ejemplo de patrón interior 0 en el grupo acentual un, dentro del enunciado La verdad es que estoy muy contento con este primer encuentro: hice un largo viaje con las interesantes y variadas informaciones pronunciado por el locutor Jordi. Las etiquetas correspondientes a los puntos de inflexión del patrón aparecen en la zona sombreada del último tier, alineadas en el tiempo con la curva original de la parte superior

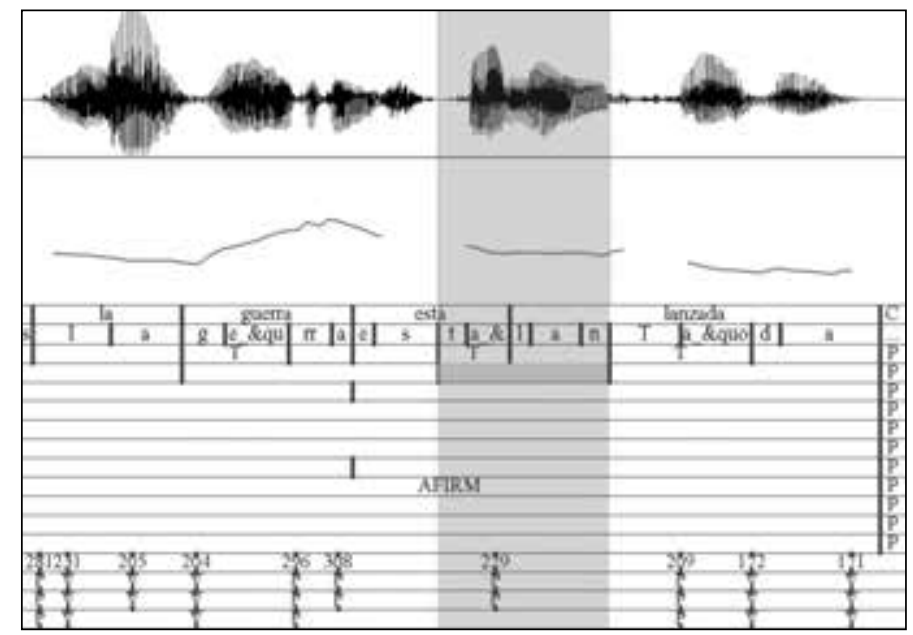

Figura 14: Ejemplo de patrón interior 0 en el grupo acentual tá lan, dentro del enunciado Así pues, la guerra está lanzada pronunciado por la locutora Mar. Las etiquetas correspondientes a los puntos de inflexión del patrón aparecen en la zona sombreada del último tier, alineadas en el tiempo con la curva original de la parte superior 


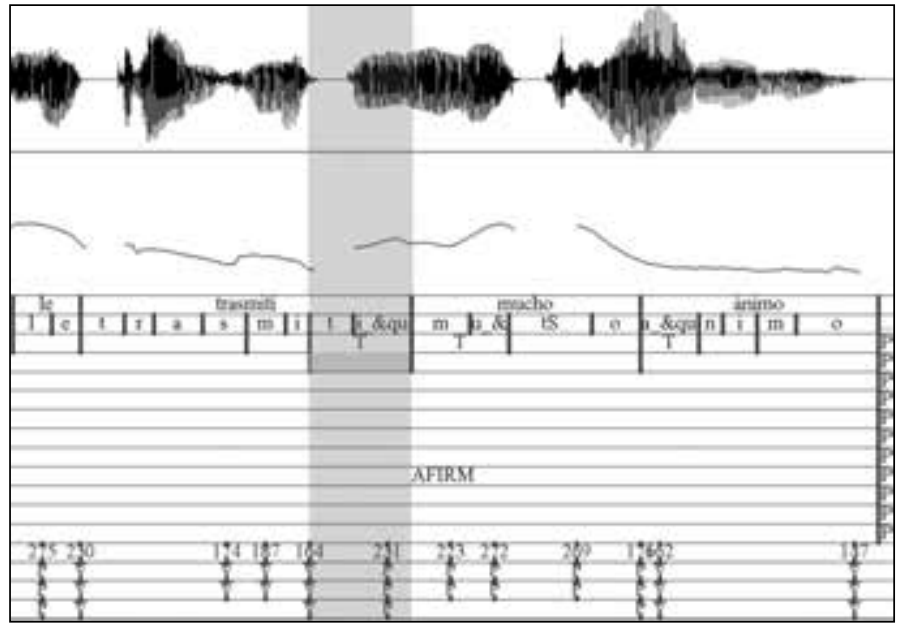

FIGURA 15: Ejemplo de patrón interior VI0_PM0 en el grupo acentual tí, dentro del enunciado Yo le transmití mucho ánimo pronunciado por la locutora Ana. Las etiquetas correspondientes a los puntos de inflexión del patrón aparecen en la zona sombreada del último tier, alineadas en el tiempo con la curva original de la parte superior

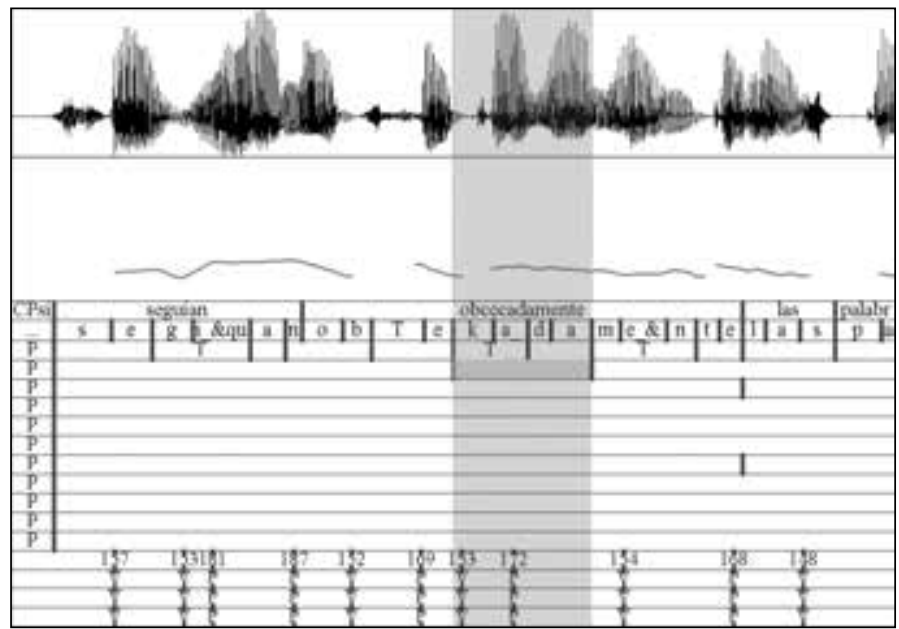

FIGURA 16: Ejemplo de patrón interior VI0_PM0 en el grupo acentual cada, dentro del enunciado Seguian obcecadamente las palabras del subprefecto pronunciado por la locutora Esm. Las etiquetas correspondientes a los puntos de inflexión del patrón aparecen en la zona sombreada del último tier, alineadas en el tiempo con la curva original de la parte superior 


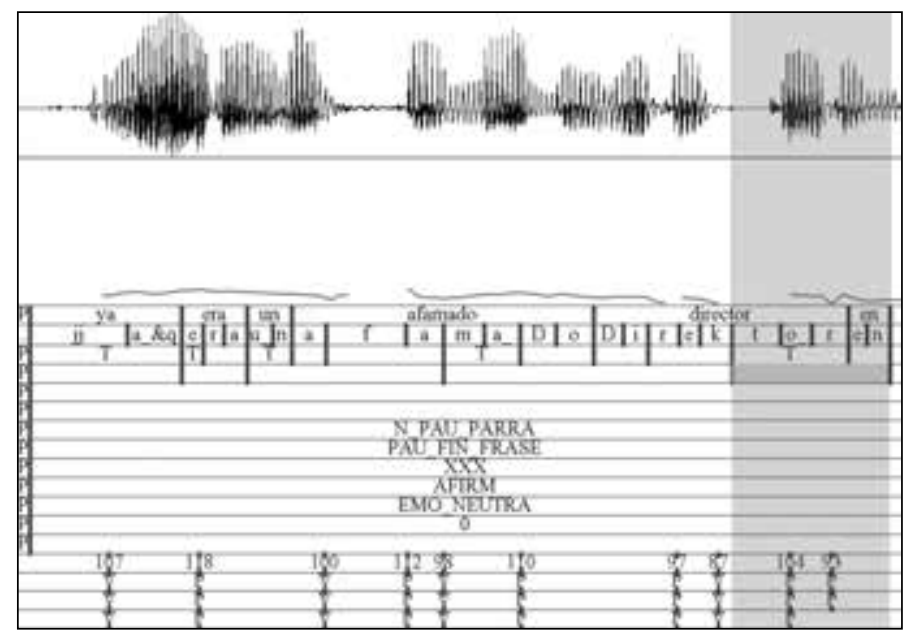

FIGURA 17: Ejemplo de patrón interior PM0 en el grupo acentual tor en, dentro del enunciado Cuando ganó un Oscar al mejor director, Gutiérrez Oliver y a era un afamado director en Gran Bretaña pronunciado por el locutor Jordi.

Las etiquetas correspondientes a los puntos de inflexión del patrón aparecen en la zona sombreada del último tier, alineadas en el tiempo con la curva original de la parte superior

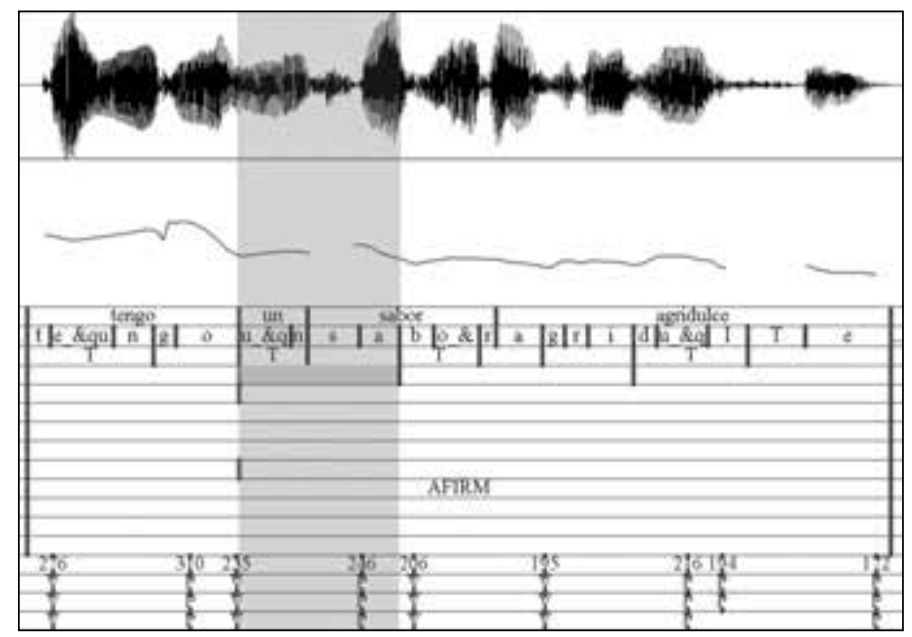

FIGURA 18: Ejemplo de patrón interior PI1 en el grupo acentual un sa, dentro del enunciado Tengo un sabor agridulce pronunciado por la locutora Mar. Las etiquetas correspondientes a los puntos de inflexión del patrón aparecen en la zona sombreada del último tier, alineadas en el tiempo con la curva original de la parte superior 


\section{Conclusiones}

A partir de los resultados presentados en el apartado anterior, se presentan ahora algunas conclusiones sobre la forma de los patrones acentuales en el corpus analizado, sus variantes y su alineamiento con las sílabas de los GA que los contienen.

A diferencia de la mayoría de descripciones anteriores, se distingue aquí entre los patrones acentuales que se dan posición inicial de grupo de los que aparecen en posición interior, porque aunque según los datos recogidos su forma presenta ciertas semejanzas (en ambas posiciones se registran variantes semejantes en lo que se refiere a la posición del pico acentual), existen también diferencias que merece la pena señalar.

En el caso de los patrones iniciales, las principales conclusiones que cabe extraer son:

1. La fuerte preferencia por los patrones con el pico en la sílaba tónica, y más concretamente en el centro del núcleo silábico, con independencia del número de sílabas del patrón y de la modalidad oracional, aunque también se dan, con menor frecuencia, los patrones con pico en la postónica. Solo en el caso de los grupos iniciales 3_1 (tres sílabas, con el acento en la primera) el patrón preferido es con el pico al inicio de la postónica (PI1). En todos los demás casos, el pico tiene tendencia a situarse preferentemente en el centro de la sílaba tónica (PM0) o, si la tónica es la primera sílaba del grupo, incluso al inicio de la misma (PI0). En otros patrones menos frecuentes, como ya se ha señalado en el apartado de resultados, el pico aparece desplazado hacia el final de la tónica (PF0) o incluso la mitad de la postónica (PM1).

2. La tendencia de los grupos iniciales (ya señalada en Garrido 1996) a comenzar con un nivel alto (P) cuando la tónica es la primera sílaba del grupo. Aunque no es sistemático (ya se ha señalado que también en esta posición el patrón VI0_PM0 se da con cierta frecuencia), sí es importante señalar que en esta posición, y en este tipo de grupos, parece darse una alternancia entre patrones con inicio bajo (V) e inicio alto (P). Esto indicaría que existe una influencia, al menos en algunos casos, del número de pretónicas iniciales en la forma del patrón.

3. Una fuerte tendencia, en los grupos donde la sílaba tónica no es la primera, a que el inicio del movimiento ascendente se sitúe al comienzo de la tónica (VI0), aunque en alguna ocasión puede avanzarse a la parte final de la sílaba pretónica (VF-1).

Así, VI0_PM0 (movimiento ascendente que comienza al inicio de la sílaba tónica y acaba en la mitad de la sílaba tónica) aparece como el movimiento más general en las tónicas iniciales del corpus analizado, con 
independencia del número de sílabas del grupo y de la posición de la tónica. A diferencia de lo que se apuntaba en algunos estudios anteriores, la tendencia dominante en el corpus analizado no es al desplazamiento del pico hacia la postónica, sino al mantenimiento dentro de la tónica.

La tabla 11 resume la forma y alineamiento tonal de los patrones más frecuentes para cada tipo de grupo acentual inicial considerado en este estudio.

\begin{tabular}{|c|c|c|c|c|}
\hline Grupo acentual & Patrón & \multicolumn{3}{|c|}{ Esquema } \\
\hline \multirow{2}{*}{$\begin{array}{l}\text { 1_1 } \\
\text { (una sílaba tónica) }\end{array}$} & PI0 & & & \\
\hline & & Tónica & & \\
\hline \multirow{2}{*}{$\begin{array}{l}2 \_1 \\
\text { (dos sílabas, } \\
\text { acento en la primera) }\end{array}$} & PI0 & & & \\
\hline & & Tónica & Postónica & \\
\hline \multirow{2}{*}{$\begin{array}{l}3 \_1 \\
\text { (tres sílabas, } \\
\text { acento en la primera) }\end{array}$} & VI0_PI1 & & & \\
\hline & & Tónica & Postónica & Postónica \\
\hline \multirow{2}{*}{$\begin{array}{l}\text { 2_2 } \\
\text { (dos sílabas, } \\
\text { acento en la segunda) }\end{array}$} & VI-1_VI0_PM0 & & & \\
\hline & & Pretónica & Tónica & \\
\hline \multirow{2}{*}{$\begin{array}{l}3 \_2 \\
\text { (tres sílabas, } \\
\text { acento en la segunda) }\end{array}$} & VI-1_VI0_PM0 & & & \\
\hline & & Pretónica & Tónica & Postónica \\
\hline \multirow{2}{*}{$\begin{array}{l}\text { 3_3 } \\
\text { (tres sílabas, } \\
\text { acento en la tercera) }\end{array}$} & VI-2_VI0_PM0 & & & \\
\hline & & Pretónica & Pretónica & Tónica \\
\hline
\end{tabular}

TABLA 11: Descripción de los movimientos básicos (forma y alineamiento tonal) identificados en este estudio para los patrones acentuales iniciales del español 
En el caso de los grupos interiores, merece destacarse especialmente:

1. El gran número de patrones con ausencia de inflexión (0) o con ausencia de pico (VI0, VM0, VF0). Esto va en contra de la idea de que el movimiento tonal típico que se asocia con las sílabas tónicas es un pico de F0, y merecería estudios posteriores más detallados.

2. En el caso de los patrones con pico, destaca la alta frecuencia de patrones con el pico en la propia sílaba tónica (PM0), aunque también se dan, en menor medida, patrones con el pico desplazado al inicio de la postónica (PI1). En este aspecto, los patrones interiores presentan un comportamiento semejante al de los patrones iniciales. Como ya se ha señalado, estos datos refuerzan la idea ya expuesta por Toledo de que el patrón acentual dominante en español es con el pico en la tónica.

3. La tendencia mayoritaria a que el inicio del movimiento ascendente anterior al pico aparezca alineado con el inicio de la sílaba tónica (VI0). Sin embargo, hay que destacar también la alta frecuencia de patrones solo con un pico al inicio (PI0) o centro de la tónica (PM0), o en la postónica (PI1), en los que el inicio del movimiento ascendente se sitúa hacia el final de la sílaba pretónica. Serían, pues, movimientos semejantes a los de los patrones VI0_PM0 y VI0_PI1, respectivamente, pero con el inicio del ascenso adelantado a la sílaba pretónica.

4. La clara preferencia por el patrón VI0_PM0 con independencia del número de sílabas postónicas que aparezcan tras la tónica en el grupo acentual. En este caso, no parece haber un efecto del número de postónicas en la forma del patrón.

Estos datos parecen indicar que los grupos iniciales e interiores comparten ciertos comportamientos. El primero es la alta frecuencia en ambos casos del patrón VI0_PM0, que se utiliza con independencia del número de sílabas del grupo y de la posición de la sílaba tónica, con lo que podría ser considerado, por su frecuencia y generalidad, el movimiento prototípico de los patrones acentuales, al menos en el corpus analizado en este trabajo. En ambos tipos de grupos, además, se registra en fenómeno del desplazamiento del pico acentual hasta la sílaba postónica (VI0_PI1), tal como ya se había descrito en trabajos anteriores, aunque con menor frecuencia que PM0. Los datos recogidos aquí no permiten extraer conclusiones acerca de los factores que determinan este desplazamiento, aunque es necesario que haya postónicas átonas para que se dé.

Cada tipo de grupo, sin embargo, presenta también sus patrones específicos. En el caso de los grupos iniciales, destaca el uso de los pa- 
trones con un nivel inicial alto (PI0, PI0_PI1) cuando la primera sílaba del grupo es la tónica. En el caso de los patrones interiores, destacan también los patrones en los que movimiento ascendente no se inicia en la misma sílaba tónica (PI0, PM0, PI1). En algunos casos, el inicio del movimiento ascendente se sitúa en la sílaba pretónica, tal como ya se había descrito anteriormente en otros trabajos.

La tabla 12 resume la forma y alineamiento tonal de los patrones más frecuentes para cada tipo de grupo acentual interior considerado en este estudio.

\begin{tabular}{|c|c|c|c|c|}
\hline Grupo acentual & Patrón & \multicolumn{3}{|c|}{ Esquema } \\
\hline \multirow{2}{*}{$\begin{array}{l}\text { 1_1 } \\
\text { (una sílaba tónica) }\end{array}$} & VI0_PM0 & & & \\
\hline & & Tónica & & \\
\hline \multirow{2}{*}{$\begin{array}{l}\text { 2_1 } \\
\text { (dos sílabas, } \\
\text { acento en la primera) }\end{array}$} & VI0_PM0 & & & \\
\hline & & Tónica & Postónica & \\
\hline \multirow{2}{*}{$\begin{array}{l}3 \_1 \\
\text { (tres sílabas, } \\
\text { acento en la primera) }\end{array}$} & VI0_PM0 & & & \\
\hline & & Tónica & Postónica & Postónica \\
\hline
\end{tabular}

TABLA 12: Descripción de los movimientos básicos (forma y alineamiento tonal) identificados en este estudio para los patrones acentuales interiores del español

\section{REFERENCIAS BIBLIOGRÁFICAS}

Beckman, M.; Díaz-Campos, M.; McGory, J. T. y Morgan, T. A. (2002): «Intonation across Spanish, in the Tones and Break Indices framework», Probus 14, pp. 9-36.

BeckMAN, M. y Pierrehumbert, J. B. (1986): «Intonational structure in English and Japanese», Phonology Yearbook 3, pp. 255-310.

Boersma, P. (2001): «Praat, a system for doing phonetics by computer», Glot International 5, 9/10, pp. 341-345.

D'Introno, F.; Del Teso, E. y Weston, R. (1995): Fonética y fonología actual del español, Madrid, Cátedra. 
Estebas, E. y Prieto, P. (2008): «La notación prosódica en español. Una revisión del Sp_ToBI», Estudios de Fonética Experimental 17, pp. 263-283.

- (2010): «Castilian Spanish intonation», en Prieto, P. y Roseano, P. (eds.), Transcription of intonation of the Spanish language, Munich, LINCOM, pp. $17-48$.

FACE, T. (2000): «A phonological analysis of rising pitch-accents in Castilian Spanish», Linguistic Symposium of Romance Languages 30, Gainesville (Florida), manuscrito.

- y Prieto, P. (2007): «Rising accents in Castilian Spanish: A revision of Sp_ToBI», Journal of Portuguese Linguistics 6/1, pp. 117-146.

FANT, L. (1984): Estructura informativa en español. Estudio sintáctico y entonativo, Upsala, Acta Universitatis Upsaliensis.

FERnÁNDEZ, A. M. y MarTínez, E. (2003): «El tono fundamental y la duración: dos aspectos de la taxonomía prosódica en dos modalidades de habla (enunciativa e interrogativa) del español», Estudios de fonética experimental 12, pp. 165-200.

GARRIDO, J. M. (1996): Modelling Spanish intonation for text-to-speech applications, Tesis doctoral, Departament de Filologia Espanyola, Universitat Autònoma de Barcelona, <http://www.tdx.cat/handle /10803/4885;jsessionid =490B02 E927B668CA56BA7A9851BDECD8.tdx1> [23/12/2011].

- (2001): «La estructura de las curvas melódicas del español: Propuesta de modelización», Lingüística Española Actual 23/2, pp. 173-210.

- (2010): «A tool for automatic F0 stylisation, annotation and modelling of large corpora», Speech Prosody 2010, Chicago, May 2010; <http://speechpro sody2010.illinois.edu/papers/100041.pdf> [23/12/2011].

—; Llisterri, J.; De la Mota, C. y Ríos, A. (1993): «Prosodic differences in reading style: Isolated vs. contextualized sentences», Eurospeech'93 Proceedings, vol. 1, pp. 573-576.

Hualde, J. (2000): «Intonation in Spanish and the other Ibero-Romance languages: Overview and status quaestionis», Linguistic Symposium of Romance Languages 30, Gainesville (Florida), manuscrito.

Jun S. -A. y Fougeron, C. (2000): «A phonological model of French intonation», en Botinis, A. (ed.), Intonation: Analysis, modelling and technology, Dordrecht, Kluwer Academic Publishers, pp. 209-242.

LlisterRi, J.; Marín, R.; De la Mota, C. y Ríos, A. (1995): «Factors affecting $\mathrm{F}_{0}$ peak displacement in Spanish», en Pardo, J. M.; Enríquez, E.; Ortega, J.; Ferreiros, J.; Macías, J. y Valverde, F. J. (eds.), Eurospeech'95. Proceedings of the 4th European Conference on Speech Communication and Technology, Madrid, 18-21 Sept. 1995, Vol. 3, pp. 2061-2064.

DE LA MotA, C. (1995): La representación gramatical de la información nueva en el discurso, Tesis doctoral, Departament de Filologia Espanyola, Barcelona, Universitat Autònoma de Barcelona.

Navarro, T. (1944): Manual de entonación española, $4^{\mathrm{a}}$ ed., Madrid, Guadarrama.

Nespor, M. y Vogel, I. (1986): Prosodic phonology, Dordrecht, Foris, Studies in Generative Grammar, 28. 
Prieto, P.; van Santen, J. y Hirschberg, J. (1994): «Patterns of F0 peak placement in Mexican Spanish», Proceedings of the Second ESCA/IEEE Workshop on Speech Synthesis, pp. 30-34.

- (1995): «Tonal alignment patterns in Spanish», Journal of Phonetics 23, pp. 429-451.

R Development Core Team (2008): $R$ : A language and environment for statistical computing, R Foundation for Statistical Computing, Viena; <http://www.R-pro ject.org $>[23 / 12 / 2011]$.

SosA, J. M. (1995): «Nuclear and pre-nuclear inventories and the phonology of Spanish declarative intonation", Proceedings of 13th ICPhS, Estocolmo, pp. 646-649.

- (1999): La entonación del español: su estructura fónica, variabilidad y dialectología, Madrid, Cátedra.

T'Hart, J.; Collier, R. y Cohen, A. (1990): A perceptual study of intonation. An experimental-phonetic approach to speech melody, Cambridge, Cambridge University Press.

Thorsen, N. (1978): «An acoustical analysis of Danish intonation», Journal of Phonetics 6, pp. 151-175.

Toledo, G. (2000): «H en el español de Buenos Aires», Langues et Linguistique 26, pp. 107-127.

- (2001): «Acentos tonales en discursos», Actas del II Congreso de Fonética Experimental, Sevilla, Universidad de Sevilla, pp. 78-88.

- (2003): «Modelo autosegmental y entonación: los corpus DIES-RTVP», Estudios de fonética experimental 12, pp. 143-163.

- (2004): «Modelo autosegmental y entonación: una muestra del corpus CREA», Revista de filología de la Universidad de La Laguna 22, pp. 313-327.

- (2007): «Acentos prenucleares: evidencia sobre el español», Language Design: Journal of Theoretical and Experimental Linguistics 9, pp. 5-34. 
